\title{
Resveratrol and red wine function as antioxidants in the central nervous system without cellular proliferative effects during experimental diabetes
}

\author{
Carina Duarte Venturini, ${ }^{1,3}$ Suélen Merlo, ${ }^{2}$ André Arigony Souto, ${ }^{4}$ Marilda da Cruz Fernandes, ${ }^{2}$ Rosane Gomez ${ }^{1,5, *}$ and Claudia \\ Ramos Rhoden' \\ 'Universidade Federal de Ciências da Saúde de Porto Alegre (UFCSPA); Division of Pharmacology; and ${ }^{2}$ Division of Histology; ${ }^{3}$ Pontifícia Universidade Católica do Rio Grande \\ do Sul (PUCRS); Institute of Geriatrics and Gerontology and ${ }^{4}$ Department of Chemistry; ${ }^{5}$ Universidade Federal do Rio Grande do Sul (UFRGS); Department of Pharmacology;
} Porto Alegre, Brazil

Key words: grape, diabetic encephalopathy, supplements, polyphenols, hippocampus, striatum, frontal cortex

Chronic hyperglycemia increases oxidative stress status and has been associated with neurological complications in diabetic individuals. This study compared the antioxidant properties of red wine or resveratrol in different brain areas of diabetic and non-diabetic rats, and investigated the effect of them on hippocampal cell proliferation in hippocampal dentate gyrus of diabetic rats. Streptozotocin-induced diabetic and control rats were treated with red wine (4 mL/ $\mathrm{kg})$, resveratrol $(20 \mathrm{mg} / \mathrm{kg}$ ) or saline, by oral gavage, for 21 days. Lipid peroxidation (TBARS), catalase and superoxide dismutase were measured to evaluate the oxidative stress and the BrdU-positive cells were assessed to measure changes in cellular proliferation. In diabetic animals, resveratrol showed antioxidant property in the hippocampus and in the striatum, while red wine had an antioxidant effect only in the hippocampus. Neither red wine nor resveratrol reversed the lower hippocampal cell proliferation in diabetic rats. Daily doses of red wine or resveratrol have an antioxidant effect in rats depending on the brain area and the glycemic status.

\section{Introduction}

Diabetes mellitus is a chronic disease characterized by metabolic disorders, in which glucose is underused, resulting in hyperglycemia. Poor glycemic control results in peripheral complications such as retinopathy, cardiopathy, nephropathy and neuropathy. ${ }^{1}$ Chronic hyperglycemia also affects the central nervous system (CNS) and the term "diabetic encephalopathy" has been used to identify structural and neurophysiologic changes in the brain, as well cognitive deficit, in diabetic patients. ${ }^{2,3}$

In peripheral tissues, the imbalance between endogenous enzymatic and nonenzymatic antioxidant mechanisms and products from glycation reactions, as a consequence of the elevated blood glucose, has been associated with cell injury in chronically hyperglycemic individuals. ${ }^{13,4}$ This condition, named oxidative stress, has also been associated also with neuronal injury in the CNS. In fact, the brain is particularly vulnerable to oxidative damage because it has abundant lipid content, a high oxygen consumption rate, and relatively scarce antioxidant enzymes compare with peripheral tissues. ${ }^{3,5}$ Chronic hyperglycemia has also been related to the impairment of hippocampal neurogenesis in diabetic animals, decreasing it by about $45 \%$ compare with non-diabetic animals. ${ }^{6}$ Lower neuronal proliferation in the hippocampus has been implicated in lower cognitive performance in rodents, mainly in memory tasks. ${ }^{7}$ Both oxidative stress and the impairment of hippocampal neurogenesis seem to contribute to the diabetic encephalopathy in chronically hyperglycemic individuals.

Exogenous antioxidants have been investigated as being potentially beneficial to decreasing or protecting against oxidative damage. ${ }^{8}$ It is already known that dietary supplementation with natural antioxidants such as lycopene, $\beta$-carotene, vitamin $\mathrm{C}$ and lipoic acid, attenuate oxidative stress and diabetic state. ${ }^{9-}$

${ }^{11}$ Recent studies have demonstrated that red wine or its natural polyphenol, resveratrol (3,5',4-trihydroxy-trans-stilbene), have a protective effect against oxidative stress in different tissues and in pathological conditions such as cardiovascular diseases, inflammatory response, cancer and diabetes. ${ }^{12}$ Resveratrol, especially abundant in grape skins and red wine, behaves as a reactive oxygen species (ROS) scavenger, metal chelator and enzyme modulator. ${ }^{13}$ Although some studies show that red wine or resveratrol decrease lipid peroxidation and increase antioxidant enzyme levels in different brain areas of diabetic rats, ${ }^{14,15}$ no study has compare the efficacy of red wine, a source of resveratrol and other compounds, with resveratrol per se, a dietetic supplement.

If diabetic encephalopathy is related to the imbalance between the production and the neutralization of ROS, and impairment in the hippocampal proliferation, prevention of oxidative stress could be considered a primary defense against development of 
complications in the CNS of diabetic individuals. Therefore, the goal of this study was to compare the antioxidant effect of a moderate consumption of red wine ( -2 glasses/day) and resveratrol supplementation in the brain of diabetic and non-diabetic rats. Additionally, we studied the effect of red wine or resveratrol on cell proliferation in the hippocampus of diabetic and non-diabetic rats.

\section{Results}

The concentration of resveratrol in red wine (Merlot varietal) was $3.2 \mu \mathrm{g} / \mathrm{mL}$. As rats were treated with $4 \mathrm{~mL} / \mathrm{kg} /$ day of red wine we can estimate that they received around 1/1,500 of the resveratrol received by rats treated with resveratrol solution $(20 \mathrm{mg} / \mathrm{kg})$, suggesting that any results from red wine are owing to its inherent properties rather than its resveratrol contend.

In this study, we initially evaluated the effect of red wine and resveratrol on oxidative stress by measuring the levels of lipid peroxidation and antioxidant enzymes activity, such as catalase (CAT) and superoxide dismutase (SOD) in the hippocampus, the frontal cortex and the striatum of chronically treated diabetic and non-diabetic rats.

In the hippocampus, both red wine and resveratrol decreased lipid peroxidation in diabetic rats $\left(\mathrm{F}_{(2,32)}=5.114 ; \mathrm{p}=0.012\right)$ (Fig. 1A). The antioxidant property of the red wine in this brain area of diabetic rats was supported by an increase of CAT activity (Fig. 1B; $\mathrm{F}_{(2,28)}=26.19 ; \mathrm{p}<0.001$ ). Resveratrol also decreased the lipid peroxidation in diabetic rats, but we did not observe concomitant changes in antioxidant enzymes (Fig. 1B and C). Resveratrol increased CAT activity only in non-diabetic rats $\left(\mathrm{F}_{(2,28)}=9.076\right.$; $\mathrm{p}<0.001$ ), supporting the idea that chronic hyperglycemia may affect the response of this enzyme in the hippocampus. Under our experimental conditions we did not see a significant increase of lipid peroxidation in the hippocampus of diabetic rats compared with non-diabetic rats.

In the frontal cortex, however, lipid peroxidation was higher in diabetic rats than in non-diabetic rats, as seen in Figure $2 \mathrm{~A}\left(\mathrm{~F}_{(1,18)}\right.$ $=15.01 ; \mathrm{p}=0.001)$. In the same figure, we show that red wine decreased the lipid peroxidation specifically for non-diabetic rats $\left(F_{(1,18)}=19.10 ; p<0.001\right)$. We could infer an antioxidant effect of the red wine, but it also decreased the SOD activity in the group of control rats (Fig. 2C; $\mathrm{F}_{(2,27)}=8.16 ; \mathrm{p}=0.007$ ). In the frontal cortex, resveratrol had antioxidant activity in both diabetic and non-diabetic rats, indicated by the lower level of lipid peroxidation (Fig. 2A; $p<0.001$ and $p=0.003$, respectively). Both, red wine or resveratrol treatment did not affect the antioxidant activity of catalase in diabetic and non-diabetic rats (Fig. 2B).

Surprisingly, our results showed that in the striatum, diabetic rats presented a lower level of lipid peroxidation compared with non-diabetic rats (Fig. 3A). One hypothesis for this result is that, in this brain area, hyperglycemia may overstimulate CAT activity (Fig. 3B; $\mathrm{F}_{(1,35)}=19.80 ; \mathrm{p}<0.001$ ) and thereby decrease the production of ROS production to levels below those found in non-diabetic rats. The other unexpected result from our study is that resveratrol presented, in this brain area, a pro-oxidant effect in diabetic rats (Fig. 3A; $\left.F_{(1,35)}=6.72 ; p=0.004\right)$. Red wine and resveratrol both had an antioxidant effect in non-diabetic rats (Fig. 3A; $\mathrm{F}_{(1,16)}=8.47 ; \mathrm{p}=0.004$ ), but only red wine increased CAT activity in these rats (Fig. 3B; $\mathrm{F}_{(1,16)}=3.83 ; \mathrm{p}=0.047$ ). In diabetic rats, red wine decreased SOD activity $\left(\right.$ Fig. $3 \mathrm{C} ; \mathrm{F}_{(2,21)}=$ $3.87 ; \mathrm{p}=0.039)$. These results point to the interference of glycemia in the response to red wine or resveratrol.

Upon comparing different brain areas we observed that CAT was expressed at a higher level in the hippocampus than in the striatum or the frontal cortex (Figs. 1B, 2B and 3B: $\mathrm{p}=0.011$ ), whereas SOD was higher in the frontal cortex than in other brain areas (Figs. 1C, 2C and 3C: $\mathrm{p}=0.006$ ) in non-diabetic rats. In diabetic rats, CAT was also higher in the hippocampus $(\mathrm{p}<0.001)$.

Our second experiment examined the effect of chronic hyperglycemia, red wine and resveratrol on cell proliferation in the hippocampus of diabetic and non-diabetic rats. BrdU is used for monitoring cell proliferation and to confirm that neurogenesis occurs in the adult mammalian brain. ${ }^{16}$ During adult neurogenesis, in the course of their maturation, newly generated neurons, astrocytes or stem cells express specific markers that reflect their state of differentiation from progenitor. ${ }^{16,17}$ Because our rats were euthanatized only $24 \mathrm{~h}$ after the first BrdU administration we cannot assume that all labeled cells in the hippocampus of our rats will be viable neurons. Our results indicated that cell proliferation in the hippocampal dentate gyrus was lower in diabetic rats, compared with other groups (Fig. 4; $\mathrm{F}_{(1,26)}=7.408 ; \mathrm{p}=0.011$ ). However, under our experimental conditions, neither red wine nor resveratrol affected cell proliferation in diabetic or non-diabetic rats.

As expected, diabetic rats lost significantly more weight than non-diabetic rats $\left(\mathrm{F}_{(1,2)}=257.0 ; \mathrm{p}<0.001\right)$ and neither red wine nor resveratrol interfered with this parameter. The average weight loss during the 3 weeks of the treatment was around $62 \pm 4 \mathrm{~g}$ for diabetic rats, whereas the control rats gained $39 \pm 5$ g. Glycemia also was higher in diabetic $(536.5 \pm 63.2 \mathrm{mg} / \mathrm{dL})$ than in nondiabetic rats $(97.1 \pm 10.9 \mathrm{mg} / \mathrm{dL})(\mathrm{p}<0.001)$, and neither red wine nor resveratrol changed this. To assure that glycemia was not affected by our dosing of red wine or resveratrol, we performed a temporal analysis of glycemia, collecting blood samples by a puncture on the distal end of the rat's tail and measuring glycemia with a glucometer every $15 \mathrm{~min}$ after 2 hours of the treatment. This test was performed in the second experiment and rats were given free access to food. There were no differences in glycemia after red wine or resveratrol treatment in diabetic and non-diabetic rats (data not show).

\section{Discussion}

In this study, we showed that daily doses of red wine and resveratrol have an antioxidant effect in rats that was dependent on the brain area and glycemic status. In diabetic rats, red wine treatment had an antioxidant effect in the hippocampus, whereas in nondiabetic rats this antioxidant effect was seen in both the frontal cortex and the striatum. In contrast, resveratrol had an antioxidant effect in the hippocampus and in the frontal cortex of diabetic rats, and in all of the studied brain areas of non-diabetic rats. 
Figure 1. The oral administration of both red wine (WINE), $4 \mathrm{~mL} / \mathrm{kg}$ or resveratrol (RES), $20 \mathrm{mg} / \mathrm{kg}$, daily for 21 days, significantly decreases the lipid peroxidation in the hippocampus of diabetic (STZ) rats, measured by malondialdehyde (MDA) levels compared to non-treated STZ rats or treated and non-treated control (CTR) rats (A, $p=0.012)$. The antioxidant property of the WINE on the hippocampus of STZ rats was confirmed by the increasing of catalase (CAT) activity $(B, p<0.001)$. Resveratrol increases the antioxidant CAT activity only in non-diabetic (CTR/ RES) rats $(B, p<0.001)$. For both CTR and STZ groups, WINE or RES do not affect the superoxide dismutase (SOD) activity $(C, p>0.05)$. Thus, red wine and resveratrol present a good profile as antioxidants in the hippocampus of rats. *Different from other treatments. Values are expressed as mean \pm SEM. $\mathrm{n}=8$ rats/ group.

It is improbable that the antioxidant effect of red wine shown here for different brain areas of diabetic and non-diabetic rats is related to its resveratrol content, since our sample of wine only contained trace amounts of this compound. Analyses of the Merlot wine used in the study confirmed that the daily ingestion of resveratrol from the wine-treated rats was $1 / 1,500$ of the amount ingested by the resveratroltreated rats. Although a large number of studies credit the antioxidant properties of red wine to the polyphenolic antioxidants present in this alcoholic beverage, ${ }^{18,19}$ other studies have shown that alcohol per se also has an antioxidant effect when administered at low to moderate doses. ${ }^{20}$ Ingestion of alcoholic beverages at low to moderate levels $24 \mathrm{~h}$ prior to ischemia and reperfusion prevents postischemic deleterious effects on neurons, a phenomenon referred to as ethanol preconditioning. ${ }^{21}$ To explain this phenomenon, authors have hypothesized that ROS formed during the first day of ethanol exposure elicit the development of a protective response in the brain that attenuates deleterious post-ischemic processes by an oxidant-dependent triggering mechanism. Thus, the apparent benefit of daily ingestion of red wine is not only related to its non-alcoholic components such as resveratrol, but also to its levels of ethanol. In fact,

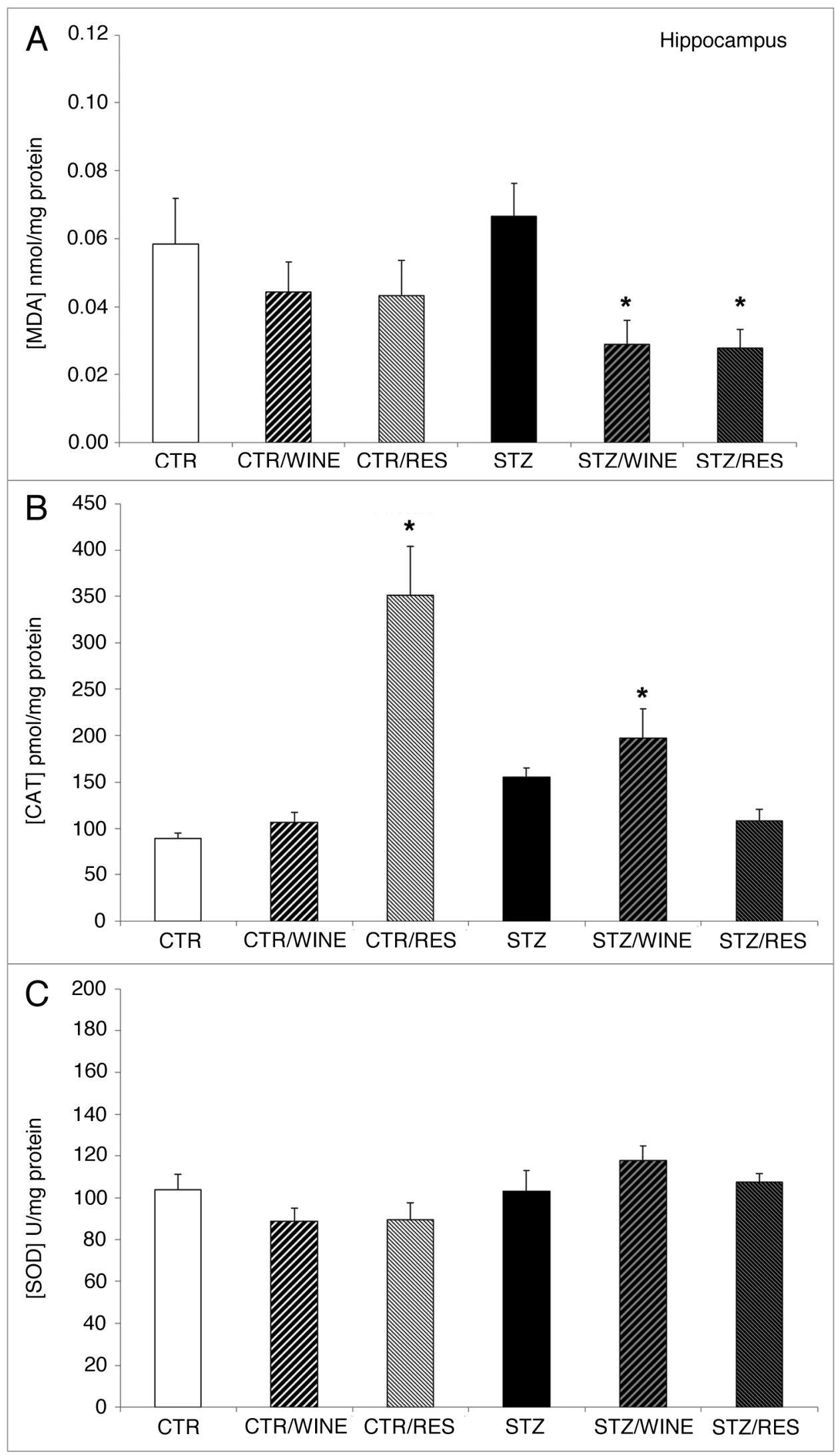

Conigrave et al. showed that frequent low-to-moderate alcohol consumption is inversely associated with risk of type 2 diabetes in men. Studies have focused their attention on isolated red wine 

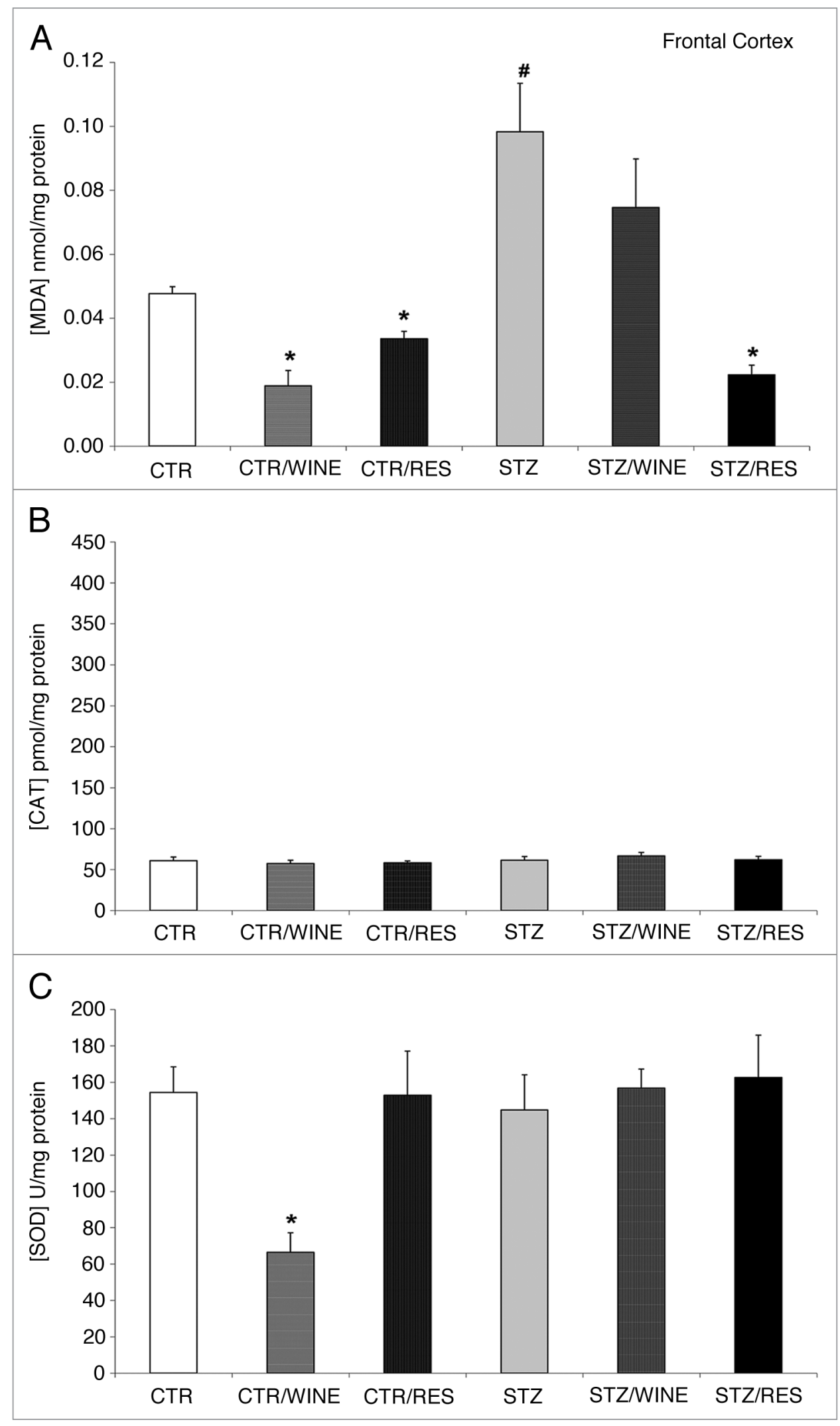

antioxidant effect in different brain areas of diabetic and non-diabetic rats.

An issue that warrants consideration is the interference of chronic hyperglycemia in the response to red wine. In the rat brain, substantial evidences supports the presence of both insulin and insulin glucose-sensitive receptors, with the highest density in the hippocampus. ${ }^{23}$ Like in the periphery, chronic hyperglycemia has been associated with neuronal insulin deficiency and/or resistance, and the development of neurodegenerative diseases in animal models. ${ }^{24,25}$ As we know from in vivo studies, glucose is elevated in the brains of diabetic rats, ${ }^{26,27}$ and we suggest that insulin resistance may interfere with the antioxidant effect of red wine, except in the hippocampus, which contains the highest density of insulin receptors. Interestingly, Carneiro et al. ${ }^{28}$ showed that Port wine, despite similar quantities of flavanols as red wine, lacks antioxidant property of wine. The authors proposed that the high content of sugars, known to have potent pro-oxidant effects, induces neuronal damage, but we cannot discard the possibility that this effect could be

antioxidants, whereas no studies have compared red wine, with its complex matrix, and resveratrol, an antioxidant polyphenol present in wine at a relatively low concentration. Our results indicate that, in spite of the trace quantity of resveratrol, red wine had an also related to insulin resistance in the brain.

In diabetic rats an unexpected result was obtained in the striatum. Rather than reducing lipid peroxidation, resveratrol increased it, suggesting, in this specific brain area, a pro-oxidant 
effect. We propose that, in the same way that the antioxidant effect of the red wine is dependent on glycemic status, resveratrol presents a pro-oxidant effect selectively in the brain regions that express a lower density of insulin receptors, such as the striatum. ${ }^{29}$ Moreover, controversial studies have shown that resveratrol decreases insulin secretion from pancreatic islets after glucose stimulation in nondiabetic rats or type 2 diabetic rats. ${ }^{30,31}$ Resveratrol does not affect basal insulin release from pancreatic islets isolated from normal rats, but clearly decreases insulin-secretory responses after glucose stimulation. ${ }^{31}$ Studies should be conducted to clarify whether this peripheral effect is reproduced in the brain and whether it may affect local insulin release in diabetic rats treated with resveratrol.

In order to explore if the antioxidant effect of red wine or resveratrol could interfere with cell proliferation we conducted an additional experiment. The result indicated that neither red wine nor resveratrol were able to reverse the reduced cell proliferation in the hippocampus of diabetic rats. This reproduced the results of previous studies, which showed that the cell proliferation rate in diabetic rats is about $40 \%$ lower compared with non-diabetic rats. ${ }^{6,32}$ Further studies will help to clarify whether higher doses of resveratrol or longer treatment would interfere with the cell proliferation in hippocampal dentate gyrus of diabetic rats. Moreover, additional experiments will be necessary to confirm the cell phenotype of those labeled-cells. Studies show that almost $75 \%$ of the surviving BrdU-positive cells express a neuronal marker, such as NeuN or neuron-specific enolase (NSE), and have physical characteristics of healthy, viable neurons. ${ }^{17}$ A small number $(-13 \%)$ of cells express a glial marker (GFAP) and the remaining cells are not labeled with either cell marker and may represent another phenotype or quiescent undifferentiated cells. ${ }^{16,17}$ As neural tissue is particularly vulnerable to ROS, we should better explore the properties of exogenous antioxidants like red wine or resveratrol in order to prevent or delay diabetic comorbidities, such as cognitive deficit, memory impairment and depression related to diabetic encephalopathy. ${ }^{13,33}$

In summary, we can conclude that, in diabetic animals, moderate daily doses of red wine have an antioxidant effect in the hippocampus and that resveratrol has an antioxidant effect in both the hippocampus and the frontal cortex. Except for the pro-oxidant effect of resveratrol in the striatum, we are inclined to state that resveratrol has a more favorable profile than red wine for diabetic individuals, as it decreases lipid peroxidation in the hippocampus and the frontal cortex. In non-diabetic rats, we cannot distinguish the effects of red wine from that of resveratrol, although red wine has a better profile in the striatum, decreasing
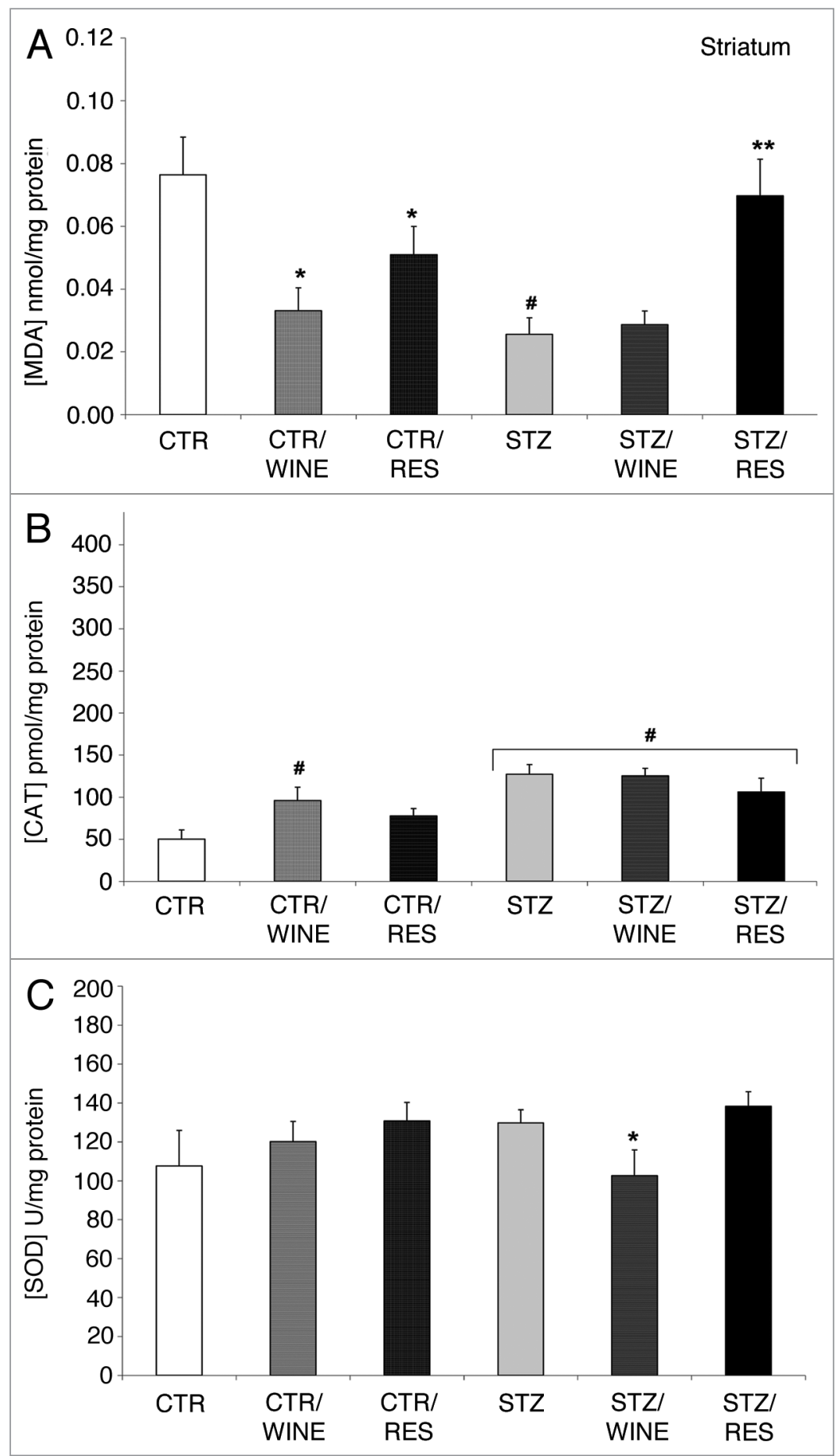

Figure 3. The oral administration of both red wine (WINE), $4 \mathrm{~mL} / \mathrm{kg}$ or resveratrol (RES), $20 \mathrm{mg} / \mathrm{kg}$, daily for 21 day, significantly decreases the lipid peroxidation in the striatum of non diabetic-rats ( $A, C T R /$ WINE and CTR/RES; $p=0.004$ ). Surprisingly, nontreated diabetic (STZ) rats present a lower lipoid peroxidation rate than non-diabetic rats (CTR), explained by the significant increasing in the antioxidant catalase (CAT) activity in the diabetic (STZ) group ( $B, p<0.001)$. Red wine increases CAT activity in $C T R$ rats $(B, p=0.047)$ and decreases the SOD activity in STZ rats $(C, p=0.039)$. RES presents, in this brain area, a pro-oxidant effect in STZ rats $(A, p=0.004)$. In this specific brain area, the antioxidant property of WINE and RES was only observed in non-diabetic animals. "Different from CTR; *Different from other treatments; ${ }^{* *}$ Different from STZ; Values are expressed as mean \pm SEM. $n=8$ rats/group. 


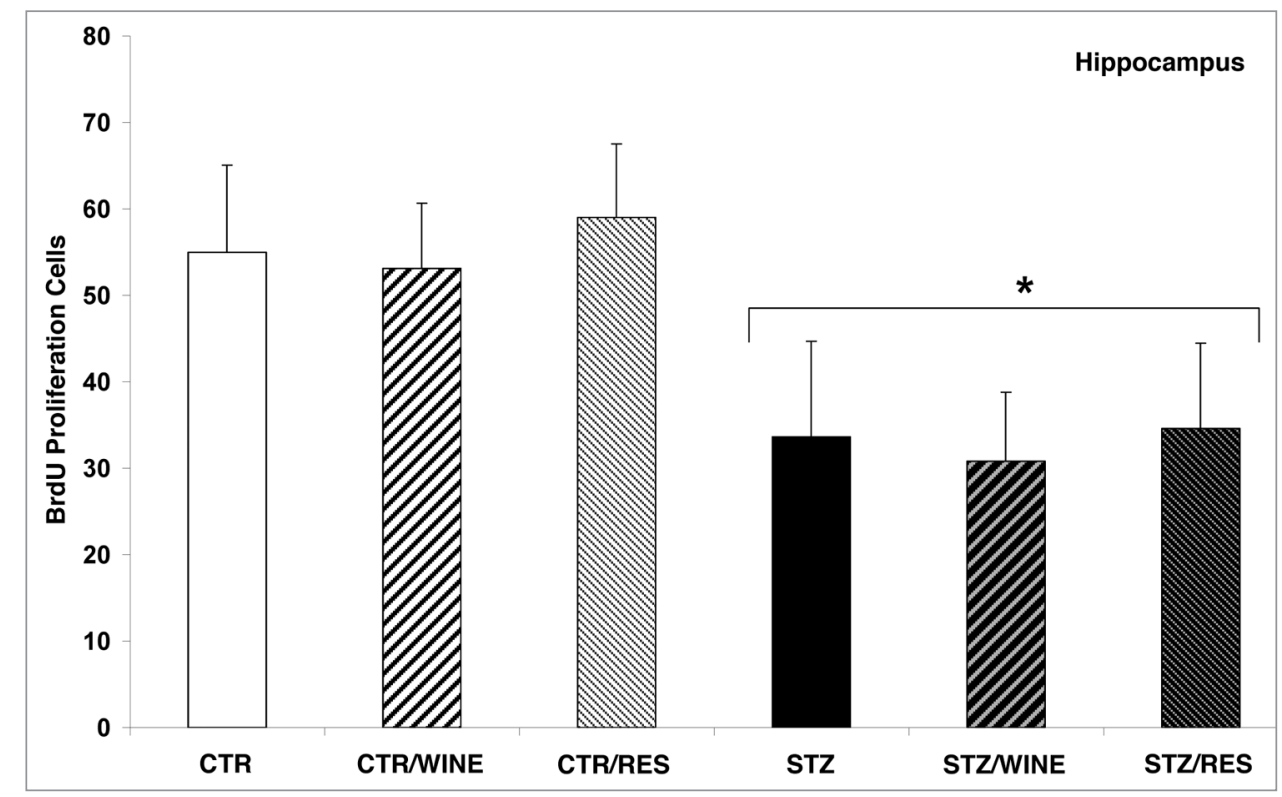

Figure 4. Although diabetic (STZ) rats present lower cellular proliferation in the hippocampal dentate gyrus than non-diabetic (CTR) rats $\left({ }^{*} p=0.011\right)$, oral administration of red wine (WINE), $4 \mathrm{~mL} / \mathrm{kg}$ or resveratrol (RES), $20 \mathrm{mg} / \mathrm{kg}$, daily for 21 day, does not stimulate cell proliferation in both diabetic and non diabetic groups. The values represent the number of BrdU-labeled cells and are expressed as mean \pm SEM, $n=8$ rats/group.

lipid peroxidation and increasing CAT activity. Further study is necessary to determine the optimum treatment with resveratrol or red wine to reverse the lower cell proliferation seen in the hippocampus of diabetic rats.

\section{Subjects and Methods}

Animals. Experiments were carried out with 96 male Wistar rats ( $\mathrm{n}=8$ /group), weighing 250-300 g. Rats were reared in the Animal Facility of Universidade Federal de Ciências da Saúde de Porto Alegre (UFCSPA) - Brazil. Rats were housed in groups of 4 in polypropylene cages under standard environmental conditions with free access to food and water. The experimental protocol was approved by the Animal Ethical Committee of UFCSPA and was conducted in accordance with the International Council for Laboratory Animal Science (ICLAS) codes.

Diabetes induction and treatments. In the first experiment, diabetes was induced in 24 animals by an i.p. injection of streptozotocin $60 \mathrm{mg} / \mathrm{Kg}$ (STZ) (Sigma Co., St. Louis, MO), dissolved in $0.05 \mathrm{M}$ citrate buffer, $\mathrm{pH}$ 4.3. The control group $(\mathrm{n}=24)$ received an equal volume of vehicle, i.p. Diabetes was confirmed with a glucometer device (Glucotrend, Boehringer Institute, Mannheim, Germany) 48 hours later and weekly until the end of the experiment. Animals with blood glucose levels $<200 \mathrm{mg} / \mathrm{dL}$ were discarded.

Immediately after diabetes status was confirmed, diabetic (STZ) and non-diabetic (CTR) groups were subdivided according to red wine (WINE; $\mathrm{n}=8$ ) (Merlot Varietal, Vinícola Miolo, Bento Gonçalves-RS, Brazil), resveratrol (RES; $\mathrm{n}=8$ ) (PanvelPharma Nostra, Porto Alegre-RS, Brazil) or saline (CTR; $\mathrm{n}=8$ ). All groups were treated via the oral route (gavage), for 3 weeks. Resveratrol was dispersed in saline with $0.05 \%$ of
Tween 80 and administered at a dose of $20 \mathrm{mg} / \mathrm{Kg}$. Red wine was administered at a volume of $4 \mathrm{~mL} / \mathrm{kg}$. The dose was calculated based on the daily intake of 2 glasses of red wine $(300 \mathrm{~mL})$ by an adult man weighting $70 \mathrm{~kg}$, containing about $0.5 \mathrm{~mL} / \mathrm{Kg} / \mathrm{day}$ of ethanol. Saline was administered $(0.9 \% \mathrm{NaCl})$ at the same volume $(4 \mathrm{~mL} / \mathrm{kg})$ to CTR and STZ control groups. All solutions were stored at $5^{\circ} \mathrm{C}$ in amber flasks, protected from light. In the last day, treatments were administered $1 \mathrm{~h}$ before euthanasia.

Oxidative stress assay. One hour from the last administration, rats were euthanatized by decapitation, and trunk blood was collected for glycemic dosage. Brain tissues were immediately dissected and quickly frozen in liquid nitrogen and individually stored at $-80^{\circ} \mathrm{C}$. When the measurements of lipid peroxidation and enzymes analysis were performed, the hippocampus, the frontal cortex and the striatum were homogenized in $120 \mathrm{mM}$ $\mathrm{KCl}$ and $30 \mathrm{mM}$ sodium phosphate buffer, $\mathrm{pH} 7.4$, containing $0.5 \mathrm{mM}$ phenylmethanesulfonyl fluoride as a protease inhibitor, at $0-4^{\circ} \mathrm{C}$. The suspensions were centrifuged at $1,000 \mathrm{xg}$ for 10 min at $0-4^{\circ} \mathrm{C}$ (Sorvall RC 5B-Rotor SM 24) and the supernatant was used for the measurements.

Determination of lipid peroxidation. Lipid peroxidation processes were determined in different brain areas by measuring thiobarbituric acid reactive species (TBARS). Homogenized tissues were precipitated with $10 \%$ of trichloroacetic acid, centrifuged, and incubated with thiobarbituric acid (Sigma, St. Louis, MO) for $60 \mathrm{~min}$ at $100^{\circ} \mathrm{C}$ and TBARS were extracted with butanol $(1: 1 \mathrm{v} / \mathrm{v})$. After centrifugation, the absorbance of the butanol was measured at $535 \mathrm{~nm}$. Malondialdehyde (MDA) standards were prepared from 1,1,3,3-tetramethoxypropane. Protein concentration in the homogenates was measured by the Bradford protein assay using bovine serum albumin as standard. 
Measurements were carried out in a Perkin Elmer Lambda 40 spectrophotometer (Shelton, CT) and results were expressed as concentration of MDA (nmol) per mg of protein.

Catalase assay. Catalase (CAT) activity was assayed with the method developed by Aebi, ${ }^{34}$ which is based on the disappearance of $\mathrm{H}_{2} \mathrm{O}_{2}$ at $240 \mathrm{~nm}$. One unit was defined as $1 \mu \mathrm{mol}$ of hydrogen peroxide consumed per min, and the specific activity was reported as units per $\mathrm{mg} /$ protein.

Superoxide dismutase assay. Superoxide dismutase (SOD) activity was assayed using the method described by Marklund. ${ }^{35}$ Cerebral tissue was homogenized 1:9 (w/v) in $50 \mathrm{mM}$ Tris- $\mathrm{HCl}$ buffer $\mathrm{pH} 8.2$ containing $1 \mathrm{mM}$ ethylenediaminetetraacetic acid (EDTA). One unit of SOD activity was defined as the amount of SOD that inhibits $50 \%$ of the pyrogallol autoxidation, and the specific activity was represented as units per mg protein.

Cell proliferation assay. In the second experiment we followed exactly the same protocol for oxidative stress evaluation as describe above, except that we administered 5-bromo-2'-deoxyuridine (BrdU) (Sigma Chemical Company, St. Louis, $\mathrm{MO}$ ), to detect and measure the S-phase of cell cycle, at 48, 24 and 2 hours before euthanasia. For this triple regime, BrdU was previously dissolved in $20 \mathrm{mg} / \mathrm{mL}$ of $\mathrm{NH}_{4} \mathrm{OH} 0.05 \mathrm{M}$ and injected at the dose of $100 \mathrm{mg} / \mathrm{kg}$, i.p. Two hours after the last BrdU administration and 1 hour after the last treatment (saline, red wine or resveratrol), rats were anesthetized with ketamine $(100 \mathrm{mg} / \mathrm{kg})$ and xylazine $(50 \mathrm{mg} / \mathrm{kg})$ i.p. and the brains were fixed by an intracardiac perfusion with $4 \%$ paraformaldeyde. After dissection, the brain was post-fixed in paraformaldeyde (4\%) followed by $70 \%$ ethanol at room temperature until it was embedded in paraffin.

For BrdU immunohistochemistry, $5 \mu \mathrm{m}$ brain sections were cut through the hippocampus, and slices were first deparaffinized $(1 \mathrm{~h}$ at $60^{\circ} \mathrm{C}$ ) and rinsed in xylol. Sections were then rinsed in phosphate-buffered saline (PBS) and blocked in bovine serum albumin (BSA). Afterward, the slices were treated with $1.5 \% \mathrm{H}_{2} \mathrm{O}_{2}$ in $0.3 \%$ methanol and washed in $0.05 \%$ Triton X-100 diluted in PBS, and then were incubated with a mouse monoclonal anti-BrdU antibody (Amersham Biosciences, 1:100) overnight at $4^{\circ} \mathrm{C}$. Subsequently, the sections were incubated with a secondary antibody peroxidase anti-mouse-IgG (Amersham Biosciences, 1.5:100) for $1 \mathrm{~h}$ at room temperature. The immunohistochemical reaction was revealed by
0.06\% 3,3-diaminobenzidine (DAB) in PBS. After being rinsed in distilled water, sections were counterstained with hematoxylin, dehydrated in ethanol and mounted on slides using Entellan ${ }^{\circledR}$.

For each brain, BrdU-positive cells were identified by their brown stain and were counted visually using an Olympus BX-40 microscope at 40x objective magnification. The total number of stained BrdU cells was determined through 10 cuts per animal with an interval of $80 \mu \mathrm{m}$ in the dentate gyrus of the hippocampus. ${ }^{36}$ Each image was analyzed twice to obtain an average labeling index.

Resveratrol concentration in the red wine. Samples from the red wine (Merlot Varietal, Vinícola Miolo, Bento Gonçalves-RS, Brazil) were collected and analyzed by high performance liquid chromatography (HPLC-Perkin-Elmer 785A), according to Souto et al. The trans-resveratrol standards were supplied by PharmaScience Inc., (Montreal, Canada), and the red wine was purchased from a commercial supplier. Before being analyzed, the wine was kept refrigerated at $4^{\circ} \mathrm{C}$, but the analyses were performed at $24^{\circ} \mathrm{C}$, and repeated 4 times. For quantification, an external standard calibration curve was done ranging from 0.10 to $10.0 \mathrm{mg} / \mathrm{L}$ of trans-resveratrol. The square regression coefficient of the analytical curve was near unity $\left(\mathrm{r}^{2}=0.9986\right)$.

Statistical analyses. Data were analyzed by a three-way ANOVA, considering diabetic condition, resveratrol or red wine treatment, and different brain areas as independent variables, and lipid peroxidation or changes in the enzyme activity as dependent variables. To evaluate the effect of the diabetes or treatments on neurogenesis we performed a two-way ANOVA test, considering diabetic condition and treatments as independent variables and stained cells in the dentate gyrus of the hippocampus as the dependent variable. Differences between groups were distinguished by the StudentNewman-Keuls post hoc test, considering $\mathrm{p}<0.05$ as an indication of statistical significance. Results were represented as mean \pm SEM.

\section{Acknowledgements}

This work was supported by Universidade Federal de Ciências da Saúde de Porto Alegre, Brazil. C.D. Venturini is supported by a fellowship from Coordenação de Aperfeiçoamento de Pessoal de Nível Superior-CAPES. CR Rhoden is supported by Conselho Nacional de Desenvolvimento Científico e Tecnológico-CNPq. We thank Vinícola Miolo Ltda, Bento Gonçalves_-RS, Brazil for providing the red wine.

\section{References}

1. Giugliano D, Ceriello A, Esposito K. Glucose metabolism and hyperglycemia. Am J Clin Nutr 2008; 87:217-22.

2. Biessels GJ, van der Heide LP, Kamal A, Bleys RL, Gispen WH. Ageing and diabetes: implications for brain function. Eur J Pharmacol 2002; 441:1-14.

3. Kikuchi S, Shinpo K, Takeuchi M, Yamagishi S, Makita Z, Sasaki N, et al. Glycation-a sweet tempter for neuronal death. Brain Res Brain Res Rev 2003; 41:306-23.

4. Yamagishi S, Matsui T. Advanced glycation end products, oxidative stress and diabetic nephropathy. Oxid Med Cell Longev 2010; 3:101-8.

5. Simonian NA, Coyle JT. Oxidative stress in neurodegenerative diseases. Annu Rev Pharmacol Toxicol 1996; 36:83-106.
6. Zhang WJ, Tan YF, Yue JT, Vranic M, Wojtowicz JM. Impairment of hippocampal neurogenesis in streptozotocin-treated diabetic rats. Acta Neurol Scand 2008; 117:205-10.

7. Jackson-Guilford J, Leander JD, Nisenbaum LK. The effect of streptozotocin-induced diabetes on cell proliferation in the rat dentate gyrus. Neurosci Lett 2000; 293:91-4.

8. Pandey KB, Rizvi SI. Plant polyphenols as dietary antioxidants in human health and disease. Oxid Med Cell Longev 2009; 2:270-8.

9. Tahrani AA, Askwith T, Stevens MJ. Emerging drugs for diabetic neuropathy. Expert Opin Emerg Drugs 2010; 15:661-83.

10. Ozsoy N, Candoken E, Akev N. Implications for degenerative disorders: antioxidative activity, total phenols, flavonoids, ascorbic acid, beta-carotene and betatocopherol in Aloe vera. Oxid Med Cell Longev 2009; 2:99-106.
11. Kuhad A, Sethi R, Chopra K. Lycopene attenuates diabetes-associated cognitive decline in rats. Life Sci 2008; 83:128-34

12. Silan C. The effects of chronic resveratrol treatment on vascular responsiveness of streptozotocin-induced diabetic rats. Biol Pharm Bull 2008; 31:897-902.

13. Harikumar KB, Aggarwal BB. Resveratrol: a multitargeted agent for age-associated chronic diseases. Cell Cycle 2008; 7:1020-35.

14. Assuncao M, Santos-Marques MJ, de Freitas V, Carvalho F, Andrade JP, Lukoyanov NV, et al. Red wine antioxidants protect hippocampal neurons against ethanol-induced damage: a biochemical, morphological and behavioral study. Neuroscience 2007; 146:158192.

15. Ates O, Cayli SR, Yucel N, Altinoz E, Kocak A, Durak $\mathrm{MA}$, et al. Central nervous system protection by resveratrol in streptozotocin-induced diabetic rats. J Clin Neurosci 2007; 14:256-60. 
16. Taupin P. BrdU immunohistochemistry for studying adult neurogenesis: paradigms, pitfalls, limitations and validation. Brain Res Rev 2007; 53:198-214.

17. Malberg JE, Eisch AJ, Nestler EJ, Duman RS. Chronic antidepressant treatment increases neurogenesis in adult rat hippocampus. J Neurosci 2000; 20:9104-10.

18. Montilla P, Espejo I, Munoz MC, Bujalance I, MunozCastaneda JR, Tunez I. Protective effect of red wine on oxidative stress and antioxidant enzyme activities in the brain and kidney induced by feeding high cholesterol in rats. Clin Nutr 2006; 25:146-53.

19. Liu L, Wang Y, Lam KS, Xu A. Moderate wine consumption in the prevention of metabolic syndrome and its related medical complications. Endocr Metab Immune Disord Drug Targets 2008; 8:89-98.

20. Crews FT, Nixon K. Mechanisms of Neurodegeneration and Regeneration in Alcoholism. Alcohol Alcohol 2009; 44:115-27.

21. Guiraud A, de Lorgeril M, Boucher F, Berthonneche C, Rakotovao A, de Leiris J. Cardioprotective effect of chronic low dose ethanol drinking: insights into the concept of ethanol preconditioning. J Mol Cell Cardiol 2004; 36:561-6.

22. Conigrave KM, Hu BF, Camargo CA Jr, Stampfer MJ, Willett WC, Rimm EB. A prospective study of drinking patterns in relation to risk of type 2 diabetes among men. Diabetes 2001; 50:2390-5.

23. Devaskar SU, Giddings SJ, Rajakumar PA, Carnaghi LR, Menon RK, Zahm DS. Insulin gene expression and insulin synthesis in mammalian neuronal cells. J Biol Chem 1994; 269:8445-54.
24. Schubert M, Gautam D, Surjo D, Ueki K, Baudler S, Schubert D, et al. Role for neuronal insulin resistance in neurodegenerative diseases. Proc Natl Acad Sci USA 2004; 101:3100-5.

25. Gerozissis K. Brain insulin, energy and glucose homeostasis; genes, environment and metabolic pathologies. Eur J Pharmacol 2008; 585:38-49.

26. Jacob RJ, Fan X, Evans ML, Dziura J, Sherwin RS Brain glucose levels are elevated in chronically hyperglycemic diabetic rats: no evidence for protective adap tation by the blood brain barrier. Metabolism 2002; 51:1522-4.

27. Gomez R, Barros HM. Clonazepam increases in vivo striatal extracellular glucose in diabetic rats after glucose overload. Pharmacol Biochem Behav 2003; 76:443-50.

28. Carneiro A, Assuncao M, De Freitas V, Paula-Barbosa MM, Andrade JP. Red Wine, but not port wine, protects rat hippocampal dentate gyrus against ethanolinduced neuronal damage-relevance of the sugar content. Alcohol Alcohol 2008; 43:408-15.

29. Unger J, McNeill TH, Moxley RT, 3rd, White M, Moss A, Livingston JN. Distribution of insulin receptor-like immunoreactivity in the rat forebrain. Neuroscience 1989; 31:143-57.

30. Su HC, Hung LM, Chen JK. Resveratrol, a red wine antioxidant, possesses an insulin-like effect in streptozotocin-induced diabetic rats. Am J Physiol Endocrinol Metab 2006; 290:1339-46.

31. Szkudelski T. The insulin-suppressive effect of resveratrol - an in vitro and in vivo phenomenon. Life Sci 2008; 82:430-5.
32. Gao Q, Gao YM. Hyperglycemic condition disturbs the proliferation and cell death of neural progenitors in mouse embryonic spinal cord. Int J Dev Neurosci 2007; 25:349-57.

33. Maiese K, Chong ZZ, Shang YC, Hou J. Novel Avenues of Drug Discovery and Biomarkers for Diabetes Mellitus. J Clin Pharmacol 2010; DOI: 10.1177/0091270010362904.

34. Aebi H. Catalase in vitro. Methods Enzymol 1984; 105:121-6.

35. Marklund S. Handbook of Methods for Oxygen radical Research. Boca Raton: CRC Press 1985.

36. Paxinos C, Watson G. The Rat Brain Stereotaxic Coordinates. New York: Academic Press 2003.

37. Souto AA, Carneiro MC, Seferin M, Senna MJH, Conz A, Gobbi K. Determination of trans-resveratro concentrations in Brazilian red wines by HPLC. J Food Comp Anal 2001; 14:441-5 


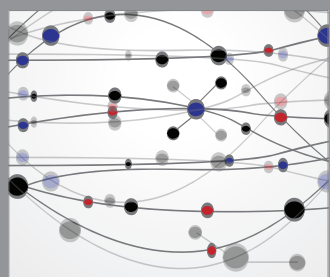

The Scientific World Journal
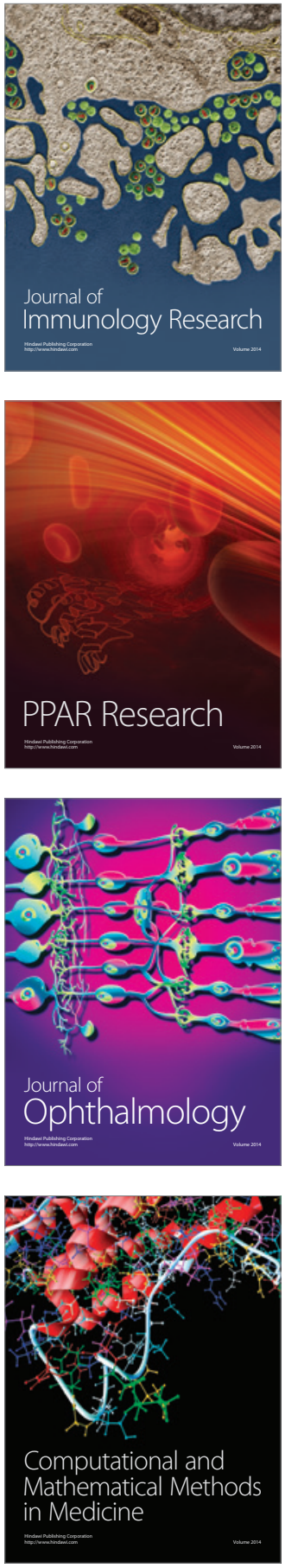

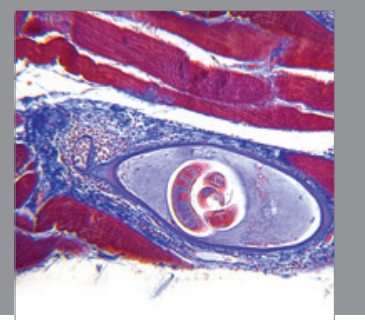

Gastroenterology

Research and Practice
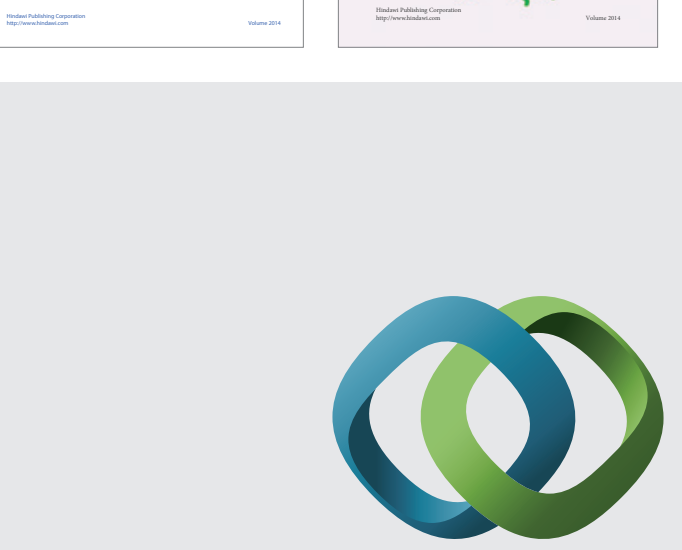

\section{Hindawi}

Submit your manuscripts at

http://www.hindawi.com
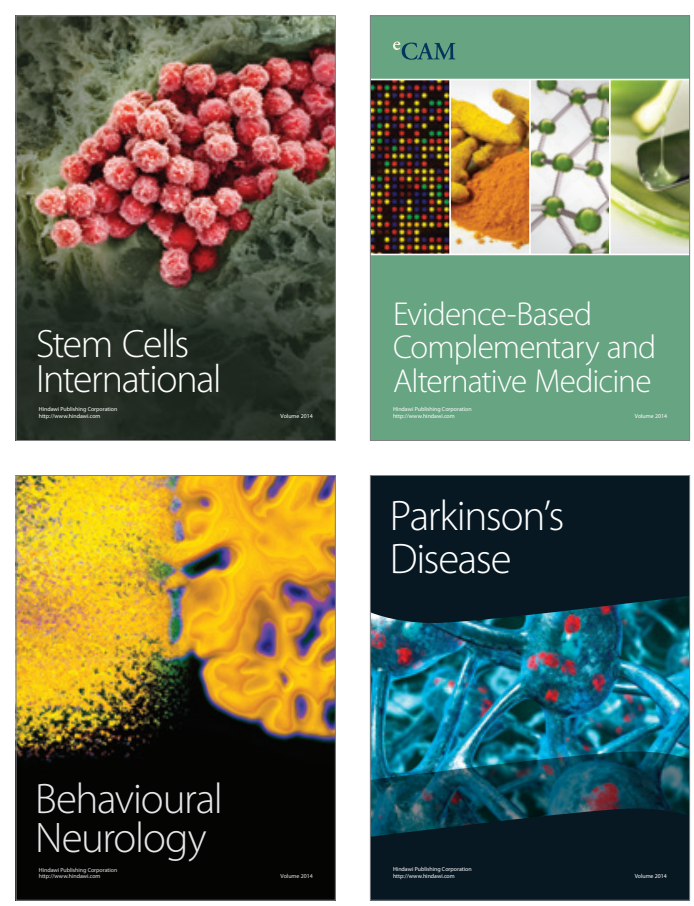

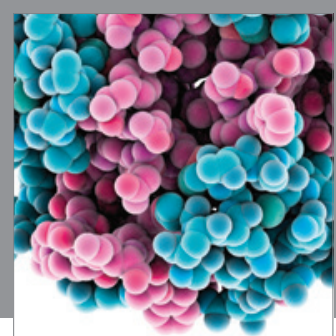

Journal of
Diabetes Research

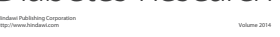

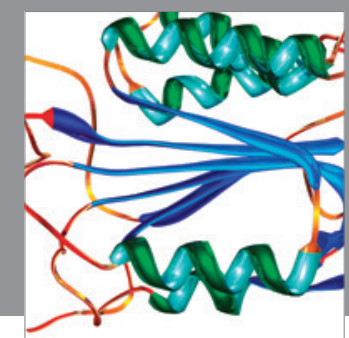

Disease Markers
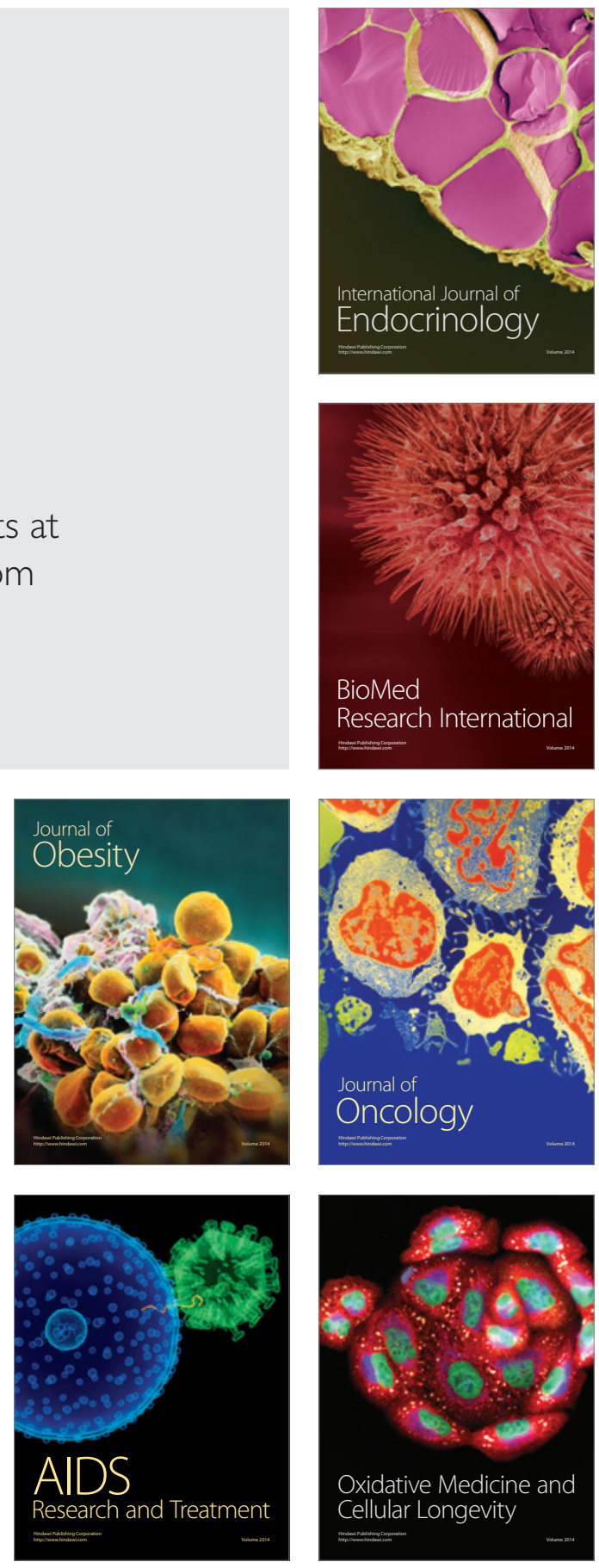